12th International Symposium on Cosmology and

Particle Astrophysics (CosPA 2015)

International Journal of Modern Physics: Conference Series

Vol. 43 (2016) 1660191 (5 pages)

(C) The Author(s)

DOI: $10.1142 / \mathrm{S} 2010194516601915$

\title{
IceCube Events from Decaying Dark Matter with Neutrino Portal
}

\author{
P. Ko and Yong Tang* \\ School of Physics, Korea Institute for Advanced Study, \\ Hoegi-ro 85, Dongdaemun-gu, Seoul 02455, Republic of Korea \\ *ytang@kias.re.kr
}

Published 7 July 2016

\begin{abstract}
IceCube has observed several $\mathrm{PeV}$ neutrino events whose astrophysical origin has not been identified. In this proceeding, we discuss heavy decaying dark matter may be responsible for these neutrinos. Dark matter $\chi$ is constructed to communicate with standard model particles through the neutrino-portal interaction. We calculate both total and differential decay width for the dominant three-body decay of dark matter and show that to fit the data, the required mass is around $\mathcal{O}(10 \mathrm{PeV})$ and lifetime is about $10^{28} \mathrm{~s}$.
\end{abstract}

Keywords: IceCube; dark matter; neutrino.

\section{Introduction}

Recently, IceCube collaboration has fully identified $3 \mathrm{PeV}$ neutrino cascade events during its 4 -year observation. ${ }^{1-5}$ One more $\mathrm{PeV}$ track event was also observed but its energy has not been determined yet. Together with other $\sim 50$ events in the energy range $\mathcal{O}(30 \mathrm{TeV})-\mathcal{O}(1 \mathrm{PeV})$, IceCube is able to claim that there is more than $6 \sigma$ significance to support the astrophysical origin of these events.

Astrophysical sources that can be responsible for these high energy neutrino events include supernova remnants, active galactic nuclei, and gamma-ray bursts. It is usually assumed that these sources inject some primary protons with specific spectra and then consider the resulting neutrino flux from proton-proton or protongamma interaction. Assuming astrophysical flux arrives isotropically with equal flavor,

$$
\Phi_{\nu}=\phi\left(\frac{E}{100 \mathrm{TeV}}\right)^{-\gamma},
$$

IceCube has given the best-fit values with three-year data,

$$
\gamma=2.5 \pm 0.09, \phi=6.7_{-1.2}^{+1.1} \times 10^{-18}[\mathrm{GeV} \mathrm{s} \mathrm{sr}]^{-1} \mathrm{~cm}^{-2} .
$$

This is an Open Access article published by World Scientific Publishing Company. It is distributed under the terms of the Creative Commons Attribution 4.0 (CC-BY) License. Further distribution of this work is permitted, provided the original work is properly cited. 
The central value for $\gamma$ has shifted to 2.58 with preliminary results based on fouryear data. With such a large index $\gamma$, although such astrophysical explanations can not be fully excluded, it is also expected that there should be accompanied with large gamma-ray flux which is highly constrained by Fermi-LAT experiment. Moreover, a large $\gamma$ indicate a smaller flux at high energy, which may not be consistent with this multi-PeV track events mentioned above.

In this contribution, we discuss a possibility that these very high energy PeV neutrino events could come from dark matter decay.

\section{Decaying Dark Matter with Neutrino Portal}

The most straightforward way to implement decaying dark matter is just to introduced a heavy right-handed neutrino, $N$, which couples to standard model particles only through the neutrino-portal interaction,

$$
\delta \mathcal{L}=y \bar{L} \tilde{H} N
$$

where $L=(\nu l)^{T}$ is a left-handed SM $S U(2)$ lepton doublet, $H$ is the SM Higgs doublet. If $y \sim 10^{-29}$ and $m_{N} \sim \mathrm{PeV}$, we might be able to produce the right amount of neutrino flux through $N \rightarrow \nu h, \nu Z, e^{ \pm} W^{\mp}$ decay channels. Since these are all twobody decay, the primary neutrino flux has $\delta$-function distribution, a sharp spectral that may not be favored by the observed neutrino events.

In our proposal, ${ }^{6}$ we have the dominant decay modes from three-body-decay which can provide a broad spectrum, see Ref. 7 for similar discussion. We consider a hidden sector with another Higgs field $\Phi$ and a Dirac fermion DM $\chi$ associated $U(1)_{X}$ gauge symmetry. Their $U(1)_{X}$ charges are assigned as follows ${ }^{\text {a: }}$ :

$$
\left(Q_{\Phi}, Q_{\chi}\right)=(1,1)
$$

We have the following renormalizable and gauge-invariant Lagrangian including just one singlet right-handed neutrino $N$ and one lepton flavor:

$$
\begin{aligned}
\mathcal{L}= & \mathcal{L}_{\mathrm{SM}}+\frac{1}{2} \bar{N} i \not \partial N-\left(\frac{1}{2} m_{N} \bar{N}^{c} N+y \bar{L} \widetilde{H} N+\text { h.c. }\right)-\frac{1}{4} X_{\mu \nu} X^{\mu \nu}-\frac{1}{2} \sin \epsilon X_{\mu \nu} F_{Y}^{\mu \nu} \\
& +D_{\mu} \Phi^{\dagger} D^{\mu} \Phi-V(\Phi, H)+\bar{\chi}\left(i \not D-m_{\chi}\right) \chi-(f \bar{\chi} \Phi N+\text { h.c. })
\end{aligned}
$$

where $X_{\mu \nu}=\partial_{\mu} X_{\nu}-\partial_{\nu} X_{\mu}$ is the field strength for $U(1)_{X}$ gauge field $X_{\mu}, F_{Y}^{\mu \nu}$ is for SM hypercharge $U(1)_{Y}$, and $\epsilon$ is the kinetic mixing parameter.

Both electroweak and $U(1)_{X}$ symmetries are spontaneously broken: $\langle H\rangle=$ $\left(0, v_{H} / \sqrt{2}\right)^{T},\langle\Phi\rangle=v_{\phi} / \sqrt{2}$. Here $v_{H} \simeq 246 \mathrm{GeV}$ is the same as SM value but $v_{\phi}$ might be taken as a free parameter. In the unitarity gauge, we replace the scalar

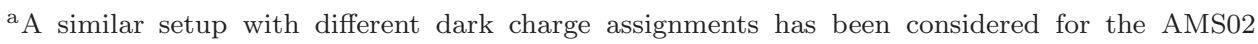
positron excess. $^{8}$
} 
fields with

$$
H \rightarrow \frac{1}{\sqrt{2}}\left(\begin{array}{c}
0 \\
v_{H}+h(x)
\end{array}\right) \text { and } \Phi \rightarrow \frac{v_{\phi}+\phi(x)}{\sqrt{2}} .
$$

When $N$ is much heavier than $\chi$, we integrate it out to obtain an effective operator,

$$
\frac{y f}{m_{N}} \bar{\chi} \Phi H^{\dagger} L+h . c .
$$

which would make $\chi$ decay possible but long-lived if $y f / m_{N}$ is sufficiently small, around $10^{-36} \mathrm{GeV}^{-1}$. This is not a problem since $y$ and $f$ is technically natural in the sense that vanishing $y$ and $f$ gives symmetries that protect $\chi$ from decay.

If $m_{\chi} \gg v_{\phi}$, the dominant decay channel is the following one, $\chi \rightarrow \phi h \nu$ :

$$
\frac{\Gamma_{3}(\chi \rightarrow \phi h \nu)}{\Gamma_{2}(\chi \rightarrow h \nu, \phi \nu)} \simeq \frac{1}{16 \pi^{2}} \frac{m_{\chi}^{2}}{v_{\phi}^{2}+v_{H}^{2}} \gg 1,
$$

because we actually have an enhancement from heavy $m_{\chi}$ even though there is a phase space suppression. Other three-body-decay channels are also equally important:

$$
\chi \rightarrow \phi / Z^{\prime}+h+\nu, \phi / Z^{\prime}+Z+\nu, \phi / Z^{\prime}+W^{ \pm}+l^{\mp},
$$

with branching ratios $1: 1: 2$ due to the Goldstone boson equivalence theorem.

\section{Numeric Results}

In Fig. 1 we show the neutrino spectra. The neutrino flux is composed of galactic and extragalactic contributions,

$$
\left.\frac{d \Phi_{\nu}^{G}}{d E_{\nu}}\right|_{E_{\nu}=E}=\left.\frac{1}{4 \pi} \sum_{i} \Gamma_{i} \int_{0}^{\infty} d r \frac{\rho_{\chi}^{G}\left(r^{\prime}\right)}{m_{\chi}} \frac{d N_{\nu}^{i}}{d E_{\nu}}\right|_{E_{\nu}=E}
$$

where $\Gamma_{i}$ is partial decay width for channel $i, d N_{\nu}^{i} / d E_{\nu}$ is the neutrino spectrum at production, $r^{\prime}=\sqrt{r_{\odot}^{2}+r^{2}-2 r_{\odot} r \cos \theta}, r$ is the distance to DM decay point, $r_{\odot} \simeq 8.5 \mathrm{kpc}$ and $\theta$ is the observation angle.

$$
\left.\frac{d \Phi_{\nu}^{E G}}{d E_{\nu}}\right|_{E_{\nu}=E}=\left.\frac{\rho_{c} \Omega_{\chi}}{4 \pi m_{\chi}} \sum_{i} \Gamma_{i} \int_{0}^{\infty} \frac{d z}{\mathcal{H}} \frac{d N_{\nu}^{i}}{d E_{\nu}}\right|_{E_{\nu}=(1+z) E},
$$

where $E^{\prime}$ is red-shifted to $E$ as $E^{\prime}=(1+z) E$, the critical energy density $\rho_{c}=$ $5.5 \times 10^{-6} \mathrm{GeV} / \mathrm{cm}^{3}$ and $\Omega_{\chi} \simeq 0.27$ is DM $\chi$ 's fraction. $\mathcal{H}$ is the Hubble parameter.

Next, we compare our model predictions with IceCube four-year data. ${ }^{5}$ To parameterize the possible astrophysical neutrino fluxes at low energy, we consider 

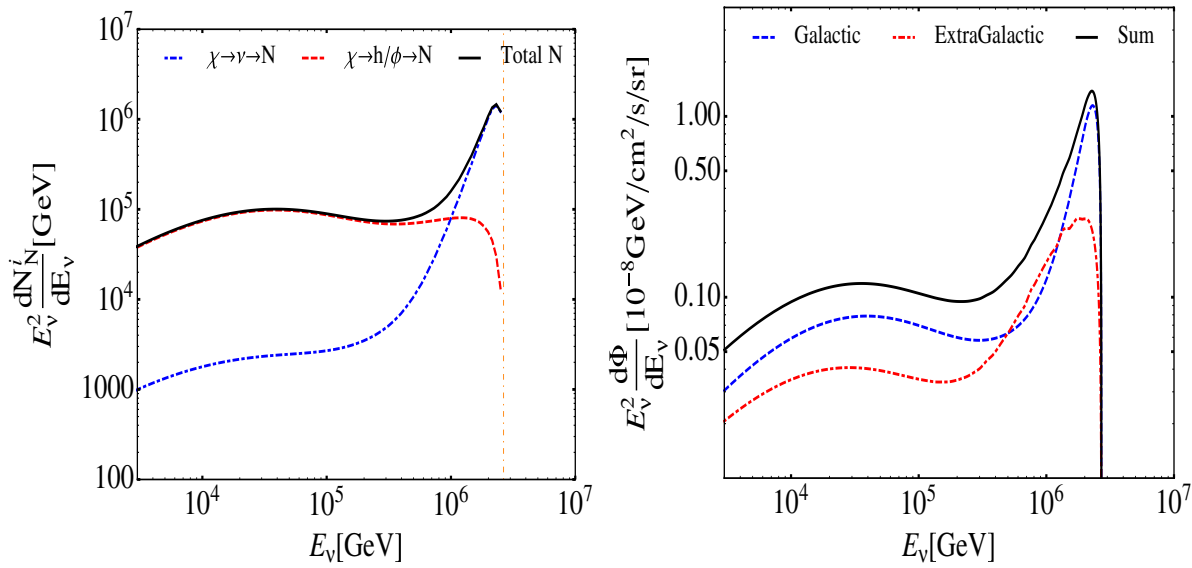

Fig. 1. Neutrino spectra from DM $\chi$ decay with $m_{\chi} \sim 5 \mathrm{PeV}$ and lifetime $\tau_{\chi}=1 / \Gamma \sim 2 \times 10^{28} \mathrm{~s}$. The left panel shows individual contribution of different final states from $\chi$ 's decay, $\nu$ and $h / \phi$, respectively. The right panel presents the galactic and extragalactic neutrino flux.
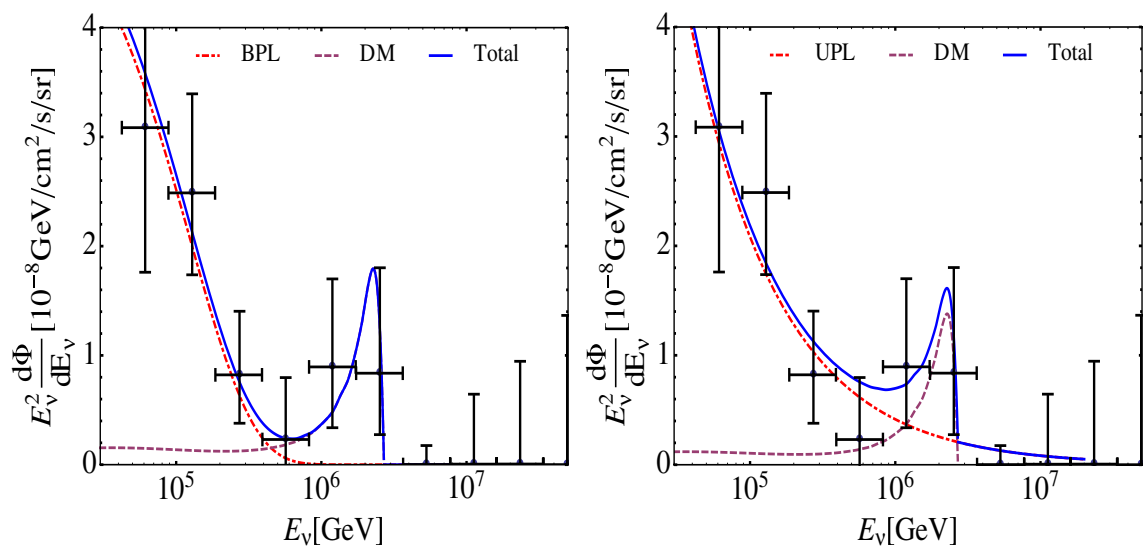

Fig. 2. Compare with preliminary results based on 4-year data, with $J_{0}^{\mathrm{BPL}}=5.6 \times$ $10^{-8} \mathrm{GeV} / \mathrm{cm}^{2} / \mathrm{s} / \mathrm{sr}$ and $\tau_{\chi} \sim 1.5 \times 10^{28} \mathrm{~s}$ (left), and $J_{0}^{\mathrm{UPL}}=2.1 \times 10^{-8} \mathrm{GeV} / \mathrm{cm}^{2} / \mathrm{s} / \mathrm{sr}$ and $\tau_{\chi} \sim 2 \times 10^{28}$ s (right).

either a broken power law (BPL) or unbroken power law (UPL),

$$
\begin{aligned}
& E_{\nu}^{2} \frac{d \Phi_{\mathrm{bkg}}}{d E_{\nu}}=J_{0}^{\mathrm{BPL}}\left(\frac{E_{\nu}}{100 \mathrm{TeV}}\right)^{-\gamma_{1}} \exp \left(-\frac{E_{\nu}}{E_{0}}\right), \\
& E_{\nu}^{2} \frac{d \Phi_{\mathrm{bkg}}}{d E_{\nu}}=J_{0}^{\mathrm{UPL}}\left(\frac{E_{\nu}}{100 \mathrm{TeV}}\right)^{-\gamma_{2}},
\end{aligned}
$$

where the first one has an exponential cut-off at energy scale $E_{0}$ which is chosen to be $125 \mathrm{TeV}$. As shown in Fig. 2, we illustrate with DM $\chi$ decay with $m_{\chi} \sim 5 \mathrm{PeV}$ and lifetime $\tau_{\chi}=1 / \Gamma \sim 2 \times 10^{28}$ s. 
We also checked that the resulting gamma-ray flux is consistent with the current astrophysical bounds.

\section{Conclusion}

We have discussed a decaying dark matter model that can explain the IceCube PeV events, based an extra $U(1)_{X}$ dark gauge symmetry and right-handed neutrino portal interactions. The dominant decay channel for dark matter $\chi$ is the threebody final state with a SM Higgs, a dark Higgs, and a neutrino $(\chi \rightarrow \phi+h+\nu)$. We show that resulting neutrino flux has a spectrum that is consistent with the IceCube observation. Together with astrophysical flux for lower energy events, we fit IceCube data around $100 \mathrm{TeV} \sim 2 \mathrm{PeV}$ if $\mathrm{DM}$ mass is about $m_{\chi} \sim 5 \mathrm{PeV}$ and its lifetime is $\tau_{\chi} \sim 2 \times 10^{28} \mathrm{sec}$.

\section{Acknowledgments}

This work is supported in part by National Research Foundation of Korea (NRF) Research Grant NRF-2015R1A2A1A05001869, by the NRF grant funded by the Korea government (MSIP) (No. 2009-0083526) through Korea Neutrino Research Center at Seoul National University, and by National Science Foundation Grant No. PHYS-1066293 (PK).

\section{References}

1. IceCube, M. Aartsen et al., First observation of PeV-energy neutrinos with IceCube, Phys.Rev.Lett. 111 (2013) 021103 [arXiv:1304.5356].

2. IceCube, M. G. Aartsen et al., Evidence for High-Energy Extraterrestrial Neutrinos at the IceCube Detector, Science 342 (2013) 1242856 [arXiv:1311.5238].

3. IceCube, M. G. Aartsen et al., Observation of High-Energy Astrophysical Neutrinos in Three Years of IceCube Data, Phys. Rev. Lett. 113 (2014) 101101 [arXiv: 1405.5303].

4. IceCube, M. G. Aartsen et al., Flavor Ratio of Astrophysical Neutrinos above 35 TeV in IceCube, Phys. Rev. Lett. 114 no. 17, (2015) 171102 [arXiv:1502.03376].

5. C. Kopper, Neutrino Astronomy, [34th International Cosmic Ray Conference].

6. P. Ko and Y. Tang, IceCube Events from Heavy DM decays through the Right-handed Neutrino Portal, Phys. Lett. B 751, 81 (2015) [arXiv:1508.02500].

7. Y. Tang, Novel Features of Gamma Ray from Dark Matter, [arXiv: 1512.03159].

8. P. Ko and Y. Tang, AMS02 positron excess from decaying fermion DM with local dark gauge symmetry, Phys. Lett. B741 (2015) 284-289 [arXiv:1410.7657]. 